\title{
Die Eintragung eines Kunstwerkes in das Verzeichnis national wertvollen Kulturgutes
}

\author{
Verwaltungsgericht Düsseldorf, Urteil vom 9. Mai 2012 - 1 K 2321/10
}

\begin{abstract}
1. Zuständig für die Eintragung eines Kunstwerkes in das „Verzeichnis national wertvollen Kulturgutes“ist die oberste Landesbehörde eines Bundeslandes in dem sich das Kunstwerk bei Inkrafttreten des Kulturgutschutzgesetzes befand und nicht dasjenige Bundesland, in dem sich das Kunstwerk oder Kulturgut zum Zeitpunkt der Einleitung des Unterschutzstellungsverfahrens befindet.
\end{abstract}

2. Bei der Ausführung von Bundesgesetzen durch die Länder nach Art. 83 GG, ist die Tätigkeit von Behörden eines anderen Landes nicht wie ein Mangel der örtlichen Zuständigkeit zu behandeln. Bei Fragen der Verbandskompetenz ist §46 VwVfG NRW nicht anwendbar. (Leitsätze der Redaktion)

\section{Tatbestand}

- Der Kläger ist der Enkel des im Jahr 1943 verstorbenen Künstlers T1.

Er wendet sich gegen die Eintragung von drei Kunstwerken T1s in das beim beklagten Land geführte Verzeichnis national wertvollen Kulturgutes.

Seit dem Tod T2s, der Ehefrau T1s, im Jahr 1987 ist der Nachlass des Künstlers Gegenstand von Auseinandersetzungen zwischen dem Kläger und der inzwischen verstorbenen Tochter des Künstlers T3 (der Mutter des Klägers) sowie T4, einer weiteren Enkelin des Künstlers.

Durch Presseberichterstattung (u.a. Frankfurter Allgemeine Zeitung, "Wenn die Erben streiten, bluten die Museen“) sowie ein Schreiben der Kulturstiftung der Länder in Berlin vom 18. November 2008 erfuhr die Staatskanzlei des beklagten Landes, dass viele Kunstwerke T1s zur Auseinandersetzung zwischen seinen oben genannten Erben bei dem Ler Auktionshaus $M$ am 6. Dezember 2008 versteigert werden sollten. In dem Schreiben der Kulturstiftung der Länder vom 18. November 2008 wurde darauf hingewiesen, dass auch drei besonders herausragende Werke des Künstlers versteigert werden sollten, die sich bisher in der Staatsgalerie in T5 bzw. dem Kunstmuseum T5 befunden hätten und unbedingt in Deutschland bleiben sollten. Hierbei handele es sich um folgende Werke:

1. $B, 1921 / 23$

2. C, 1919

3. $\mathrm{T}, 1935$.

Dem Schreiben, dass mit der Bitte um Prüfung schloss, ob diese drei bereits von T5 zur Versteigerung nach $L$ verbrachten Kunstwerke in das Gesamtverzeichnis national wertvollen Kulturgutes eingetragen werden könnten, war u.a. ein „Gutachten zum Antrag auf Unterschutzstellung in das Gesamtverzeichnis national wertvollen Kunstgutes" vom 13. November 2008 von
Frau N beigefügt, der Verfasserin des Werkverzeichnisses T1s. Das Gutachten geht - in unterschiedlichem Umfang - auf die Werke selbst, die Umstände ihrer Entstehung, die angewandten Techniken und ihre Bedeutung für das Gesamtwerk des Künstlers ein.

Nach einem Aktenvermerk vom 24. November 2008 entschied die Staatskanzlei an diesem Tag, ein Unterschutzstellungsverfahren nach dem Gesetz zum Schutz deutschen Kulturgutes gegen Abwanderung vom 6. August 1955 (neugefasst durch Bekanntmachung vom 8. Juli 1999, BGBI. I, S. 1754) - Kulturschutzgesetz - (KultgSchG) einzuleiten. In dem Vermerk heißt es, das Verfahren solle wegen der in Kürze erfolgenden Versteigerung zunächst auf die drei genannten Werke beschränkt werden; möglicherweise würden später weitere Kunstwerke T1s in das Verfahren einbezogen. Hinsichtlich dieser drei Werke lägen ausreichende Anhaltspunkte dafür vor, dass ihre Abwanderung aus dem Bundesgebiet einen wesentlichen Verlust für den deutschen Kulturbesitz bedeuten würde.

Mit Schreiben vom 1. Dezember 2008 informierte die Staatskanzlei das Auktionshaus $M$ über die Verfahrenseinleitung und das sich aus $\S 4$ Abs. 1 KultgSchG ergebende Ausfuhrverbot. Mit Schreiben vom 3. Dezember 2008 wurden der Kläger, seine Mutter sowie T4 über die Verfahrenseinleitung und die beabsichtigte Eintragung der Werke in das Verzeichnis national wertvollen Kulturgutes informiert. Ihnen wurde Gelegenheit zur Stellungnahme bis zum 20. Januar 2009 gegeben.

Durch eine von der Mutter des Klägers erwirkte einstweilige Verfügung des Oberlandesgerichts München vom 3. Dezember 2008 (Az. 18 U 5329/08) wurde die für den 6. Dezember 2008 beabsichtigte Versteigerung untersagt und die Herausgabe der Kunstwerke an einen Sequester angeordnet, wo sie sich nach den von den Beteiligten in der mündlichen Verhandlung gemachten Angaben offenbar noch immer befinden.

Mit Schreiben vom 10. Dezember 2008 forderte die Staatskanzlei gemäß § 2 Abs. 2 Satz 1 und 2 KultgSchG fünf Mitglieder des von ihr berufenen Sachverständigenausschusses auf, ihre Stellungnahme zu der beabsichtigten Eintragung der drei Kunstwerke in das Verzeichnis national wertvollen Kulturgu- 
tes abzugeben. Drei der fünf Sachverständigen kamen zu der Einschätzung, dass alle drei Kunstwerke eingetragen werden sollten. Ein Sachverständiger lehnte die Eintragung insgesamt ab, ein weiterer befürwortete sie nur hinsichtlich des Werkes „B, $1921 / 23^{\prime \prime}$

Am 18. Dezember 2008 wurde die Einleitung des Unterschutzstellungsverfahrens im Bundesanzeiger bekannt gemacht.

Mit Schreiben an die Staatskanzlei vom 14. Januar 2009 zeigte der Prozessbevollmächtigte des Klägers - ohne Vorlage einer Vollmacht - an, den Kläger und dessen Mutter zu vertreten und kündigte eine Stellungnahme an.

Unter dem 31. März 2009 nahm er dann für die Mutter des Klägers unter Vorlage einer entsprechenden Vollmacht Stellung und führte aus, der Ministerpräsident des Landes NordrheinWestfalen sei für das Unterschutzstellungsverfahren nicht zuständig, weil nach $\S 1$ Abs. 1 Satz 1 KultgSchG Kunstwerke in dem Land, in dem sie sich bei Inkraftreten des Kulturschutzgesetzes befinden, in ein „Verzeichnis national wertvollen Kulturgutes" einzutragen seien. Bei Inkrafttreten des Kulturschutzgesetzes seien die fraglichen Kunstwerke - unabhängig davon, ob man auf die ursprüngliche Gesetzesverkündung vom 6. August 1955 oder auf die Neubekanntmachung vom 8. Juli 1999 abstelle - zwar in Deutschland, aber nicht in Nordrhein-Westfalen gewesen. Dass die Kunstwerke jetzt zur Versteigerung nach $\mathrm{L}$ verbracht worden seien, begründe die Zuständigkeit des beklagten Landes nicht; ein solches allgemeines Belegenheitsprinzip lasse sich dem Kulturschutzgesetz nicht entnehmen. Allenfalls dauerhaften Ortswechseln könne in Anknüpfung an $\S 9$ Abs. 3 KultgSchG insoweit Bedeutung zukommen. Hier seien die Kunstwerke aber nur zu einem vorübergehenden Zweck nach $L$ gelangt. In diese Richtung weise auch die unter dem 22 . April 2004 beschlossene Empfehlung der Kultusministerkonferenz zur Eintragung von Kulturgütern in das Verzeichnis national wertvollen Kulturgutes, wonach $\S 1$ Abs. 1 Satz 1 KultgSchG als Zuständigkeitsregelung aufzufassen sei. In materiell-rechtlicher Hinsicht lägen die Voraussetzungen für eine Eintragung der fraglichen drei Kunstwerke nicht vor. Die Abwanderung der Kunstwerke würde nicht zu einem wesentlichen Verlust für den deutschen Kulturbesitz führen, wie es $\S 1$ Abs. 1 Satz 1 KultgSchG verlange. Bei der Beantwortung der Frage, ob ein Kunstwerk "national wertvoll" sei, sei von einem objektbezogenen Ansatz auszugehen, weshalb es nicht allein genüge, dass es sich um Bilder eines auch international hochrangigen Künstlers handele. Die Kunstwerke müssten gerade auch innerhalb des Gesamtwerkes des Künstlers eine herausragende Stellung einnehmen. Dass dies bei den Kunstwerken, auf die sich das Eintragungsverfahren beziehe, der Fall sei, lasse sich aber weder dem Gutachten von N noch den Schreiben der Staatskanzlei entnehmen. Das Gutachten beschränke sich auf eine weitgehend werkimmanente Beschreibung. Zudem sei $\mathrm{N}$, auch wenn sie eine hervorragende Kennerin des Fuvre von T1 sei, nicht unparteiisch, denn als frühere Vizedirektorin der Staatsgalerie
T5 sei sie geneigt, die Interessen der Staatsgalerie und anderer T5er Museen tatkräftig zu unterstützen, den Bestand an Werken von $\mathrm{T} 1$ zu erhalten bzw. zu erwerben. Es sei auch nicht ersichtlich, weshalb angesichts des vergleichsweise kleinen bildhauerischen Fuvre von T1 gleich zwei seiner Plastiken unter Schutz gestellt werden sollten. Von dem Kunstwerk "C, 1919“ gebe es zudem weitere originale wie auch posthume Abgüsse. Was die Bedeutung gerade des in $\mathrm{L}$ befindlichen Gipsabgusses ausmache, sei nicht klar. Insgesamt werde die Bedeutung der beiden Plastiken überbewertet.

Der "T" stehe - anders als die beiden Plastiken - nicht im Gesamthandseigentum der Erbengemeinschaft T2, sondern gehöre allein dem Kläger. Diesem sei er von seiner Mutter geschenkt worden, die ihn wiederum von ihrer Mutter - der Ehefrau T1s - nach dessen Tod im Wege der Schenkung erhalten habe. Das Bild sei nie ausgestellt oder Gegenstand von Leihanfragen gewesen. Auch in Veröffentlichungen über das Werk T1s komme ihm nur eine untergeordnete Bedeutung zu, weshalb in Bezug auf dieses Bild die Eintragungsvoraussetzungen nicht vorlägen.

Schließlich verfolge die Staatskanzlei mit dem Eintragungsverfahren den gesetzeswidrigen Zweck, den Verkauf der Werke an ausländische Bieter zu hohen Preisen zu verhindern und so den T5er Museen den Erwerb zu ermöglichen. Der Rat der Stadt T5 habe am 3. Dezember 2008 beschlossen, dass das Bild "T, 1935" bei der Versteigerung angekauft und hierfür ein Betrag von 800.000 Euro zur Verfügung gestellt werden solle.

In seinem Schreiben vom 31. März 2009 gab der Kläger an, sich den Ausführungen des Rechtsanwaltes seiner Mutter anzuschließen, und vertiefte einige der vorgenannten Argumente.

Mit inhaltsgleichen Bescheiden an den Kläger, seine Mutter und Frau T4 vom 26. Februar 2010 verfügte der Ministerpräsident des Landes Nordrhein-Westfalen die Eintragung der fraglichen, in der Verfügung näher bezeichneten Kunstwerke T1s in das Verzeichnis national wertvollen Kulturgutes des beklagten Landes. Zur Begründung führte er aus, die Eintragung der Kunstwerke in das Verzeichnis national wertvollen Kulturgutes habe zwingend erfolgen müssen, weil die Abwanderung der Werke einen wesentlichen Verlust für den deutschen Kulturbesitz bedeuten würde und der Behörde bei ihrer Entscheidung weder ein Beurteilungsspielraum noch Ermessen zustünde.

Die Zuständigkeit des Ministerpräsidenten des Landes Nordrhein-Westfalen sei gegeben, weil sich die Bilder bei Einleitung des Unterschutzstellungsverfahrens in Nordrhein-Westfalen befunden hätten. Aus der Formulierung des $\S 1$ Abs. 1 Satz 1 KultgSchG folge nicht, dass spätere Ortswechsel keine Auswirkungen auf die Zuständigkeit für die Entscheidung über die Eintragung hätten. Ein strenges Verständnis des § 1 Abs. 1 Satz 1 KultgSchG liefe dem Gesetzeszweck zuwider, die Abwanderung national wertvollen Kulturgutes effektiv zu verhindern. Bei einer solchen Sichtweise müssten zunächst oft aufwändige Ermittlungen über den Aufenthaltsort des Kunstwerkes bei In- 
krafttreten des Kulturgutschutzgesetzes angestellt werden. Die weiteren Regelungen in $\S \S 1$ Abs. 2, 8 und 9 KultgSchG hülfen nicht weiter, weil sie nur bereits eingetragenes Kulturgut beträfen. Auch die Empfehlung der Kultusministerkonferenz in deren Beschluss vom 22. April 2004 stünde dieser Sichtweise nicht entgegen, denn die Kultusministerkonferenz habe dadurch, dass sie § 1 Abs. 1 Satz 1 KultgSchG als Zuständigkeitsregelung und nicht als zeitliche Sperre bezeichnet habe, lediglich zum Ausdruck bringen wollen, dass die Regelung keine zeitliche Zäsur bei der Anwendbarkeit des Gesetzes bewirke. Welches Bundesland bei der nachträglichen Verbringung eines Kunstwerkes in das Gebiet eines anderen Bundeslandes zuständig sei, habe nicht geklärt werden sollen.

In materieller Hinsicht komme es unabhängig vom Ergebnis der Sachverständigenanhörung und vom Inhalt etwaiger Gutachten allein darauf an, ob objektiv die Eintragungsvoraussetzungen vorlägen. Aus diesem Grund müsse den N gegenüber geäußerten Vorbehalten nicht weiter nachgegangen werden. Jedenfalls seien die fraglichen Kunstwerke objektiv als national wertvoll zu qualifizieren. Mit allen drei Werken seien die Phasen der künstlerischen Entwicklung T1s paradigmatisch belegt und anschaulich nachvollziehbar. Sie zeigten seine übergreifenden Tätigkeiten als Wandgestalter, Plastiker, Zeichner und Maler, der sich Zeit seines Lebens um ein Gesamtkunstwerk bemüht habe. Die drei Werke dokumentierten T1s Bedeutung als Begründer des figürlichen Konstruktivismus. T1 thematisiere die Stellung der menschlichen Figur im Raum und belege die technisch funktionale Interpretation seines Menschenbildes als Gegenthese zur reinen Kreatürlichkeit mit besonderer Anschaulichkeit. Im Anschluss gehen die Bescheide auf die einzelnen Werke betreffende Gesichtspunkte ein. Die Bescheide wurden Frau T4 sowie dem Verfahrensbevollmächtigten der zu diesem Zeitpunkt bereits verstorbenen Mutter des Klägers am 3. März 2010 gegen Postzustellungsurkunde zugestellt. Der als internationales Einschreiben an die Anschrift des Klägers in Italien gesandte Bescheid konnte nicht zugestellt werden.

Der Kläger hat am 6. April 2010 Klage erhoben.

Er trägt vor, ihm sei die angegriffene Entscheidung des Ministerpräsidenten des Landes Nordrhein-Westfalen zwar nicht bekanntgegeben worden. Diese sei aber dennoch durch die Zustellung an den damaligen Verfahrensbevollmächtigten seiner zum Zeitpunkt der Zustellung bereits verstorbenen Mutter wirksam geworden. Seine Klagebefugnis sei gegeben, weil er der einzige Nachkomme seiner Mutter sei; das Bild "T, 1935" stehe in seinem Alleineigentum.

In der Sache hält er die angegriffene Entscheidung für rechtswidrig. Hierzu wiederholt und vertieft der Kläger die bereits im Verwaltungsverfahren vorgebrachten Argumente. Zudem sei es auch in tatsächlicher Hinsicht zweifelhaft, ob das Kunstwerk „B, 1921/23“ zum Zeitpunkt der Verfahrenseinleitung bereits in $L$ gewesen sei. Seit dem Inkrafttreten des Kulturgutschutzgesetzes hätten sich die verfahrensgegenständlichen Kunstwerke
T1s ununterbrochen in Baden-Württemberg befunden. Nur für nach Inkrafttreten des Kulturgutschutzgesetzes geschaffene oder in den Geltungsbereich des Gesetzes gelangte Kunstwerke mache es Sinn, auf die Belegenheit zum Zeitpunkt der Verfahrenseinleitung abzustellen. Die gesetzliche Regelung erlaube auch durchaus einen effektiven Schutz abwanderungsgefährdeter Kunstwerke, denn sowohl hier als auch in anderen Fällen seien die Standorte der jeweiligen Kunstwerke bekannt und eindeutig gewesen. Das beklagte Land stütze sich nur auf Praktikabilitätserwägungen und nicht auf dogmatische Argumente und stelle hierbei die Sinnhaftigkeit des eindeutigen Gesetzeswortlauts in Frage. Die Schaffung einer der Vorgehensweise des beklagten Landes entsprechenden gesetzlichen Regelung sei aber dem Gesetzgeber vorbehalten, der die fragliche Bestimmung bei der Neubekanntmachung des Kulturgutschutzgesetzes im Jahr 1999 nicht geändert habe. Zwar habe das Verwaltungsgericht Berlin in seinem Urteil vom 29. November 2006 (Az. 1 A 162.05) einem späteren Ortswechsel bezüglich der Eintragungszuständigkeit Bedeutung beigemessen. Hierbei habe es sich allerdings um einen auf Dauer angelegten Ortswechsel gehandelt. Das Handeln des beklagten Landes stelle nicht nur einen Mangel der örtlichen Zuständigkeit, sondern zudem einen nicht heilbaren Verstoß gegen die Verbandskompetenz dar.

\section{Der Kläger beantragt,}

die Entscheidung des Ministerpräsidenten des Landes Nordrhein-Westfalen über die Eintragung der Kunstwerke 1. C, 1919, 2. T, 1935, 3. B, 1921/23 des Künstlers T1 vom 26. Februar 2010 aufzuheben.

Das beklagte Land beantragt,

die Klage abzuweisen.

Es verweist zur Begründung auf die angegriffene Entscheidung des Ministerpräsidenten des Landes Nordrhein-Westfalen vom 26. Februar 2010 und führt ergänzend aus, dass effektiver Schutz national wertvollen Kulturgutes nicht gewährleistet werden könne, wenn vor Einleitung des Eintragungsverfahrens stets geprüft werden müsste, wo sich das Kulturgut am 6. August 1955 befunden habe. Aus gesetzgeberischer Sicht sei die Frage der Zuständigkeit auch weniger wichtig, weil die Entscheidung über die Eintragung gebunden und im gesamten Bundesgebiet einheitlich ausgestaltet sei. Mit § 1 Abs. 1 Satz 1 KultgSchG habe der Gesetzgeber nicht den effektiven Kulturgutschutz erschweren, sondern nur seiner Erwartung an die Exekutive Ausdruck verleihen wollen, die fortgeschrittene Abwanderung national wertvollen Kulturgutes durch eine zeitnahe Eintragung zu stoppen. Mit Rücksicht auf die Effizienz des bezweckten Kulturgutschutzes könne es auch nicht darauf ankommen, ob sich ein national wertvolles Kulturgut dauerhaft oder nur vorübergehend in einem bestimmten Bundesland befinde. 
Aber selbst wenn das beklagte Land für die angegriffene Entscheidung nicht zuständig gewesen sein sollte, stehe die Regelung des $\S 46$ Verwaltungsverfahrensgesetz für das Land Nordrhein-Westfalen (VwVfG NRW) der Aufhebung des Bescheids entgegen. Hier sei offensichtlich, dass die behauptete Verletzung von Vorschriften über die örtliche Zuständigkeit die Entscheidung in der Sache nicht beeinflusst habe, denn bei der Entscheidung über die Eintragung in das Verzeichnis national wertvollen Kulturgutes handele es sich um eine gebundene Entscheidung. Eine fehlende Zuständigkeit des beklagten Landes beträfe allein die Frage der örtlichen Zuständigkeit und nicht der Verbandskompetenz. Zudem sei dem Wortlaut des $\S 46$ VwVfG NRW eine solche Einschränkung seines Anwendungsbereichs fremd. Auch sei zur Zeit der Entstehung des Kulturgutschutzgesetzes die rechtsdogmatische Klärung von Zuständigkeitsfragen noch nicht so weit wie heute fortgeschritten gewesen. Allgemein sei bei der Ausführung von Bundesgesetzen durch die Länder nach Art. 83 Grundgesetz (GG) die Tätigkeit von Behörden eines anderen Landes wie ein Mangel der örtlichen Zuständigkeit zu behandeln. Dies entspreche auch dem Sinn der verfahrensökonomischen Zwecken dienenden Vorschrift des § 46 VwVfG NRW. Richtige Sachentscheidungen sollten nicht wegen eines Verfahrensfehlers aufgehoben werden, wenn und weil sie sogleich nach materiellem Recht wieder erlassen werden müssten. Nach dem Urteil des OVG Hamburg vom 16. Februar 1999 (Az. Bf VI 2/97) komme der Einhaltung des Kompetenzbereichs im Verhältnis zu einem anderen Bundesland in Verfahren, in denen die Bundesländer Bundesgesetze als eigene Angelegenheiten ausführen, wegen der Einheitlichkeit des Verfahrensrechts regelmäßig keine eigenständige, über den Mangel der örtlichen Zuständigkeit hinausreichende Bedeutung zu. Es finde kein Übergriff in eine Zuständigkeit statt, die im Verhältnis zweier Körperschaften zueinander prinzipiell der anderen Körperschaft vorbehalten sei. Der unter dem Gesichtspunkt der Verbandskompetenz spezifisch geschützte Eigenbereich sei zumal dann nicht wesentlich berührt, wenn das Verwaltungshandeln im Vollzug des Bundesgesetzes wie hier ohne Ermessen strikt gebunden sei.

Das Unterschutzstellungsverfahren sei auch nicht willkürlich auf die fraglichen drei Kunstwerke beschränkt worden. Ausweislich des Vermerks der Staatskanzlei vom 24. November 2008 sei das Verfahren wegen der aus damaliger Sicht kurzfristig anstehenden Versteigerung zunächst auf die drei Werke beschränkt worden. $\mathrm{Ob}$ an diesen drei Kunstwerken ein Erwerbsinteresse T5er Museen bestanden habe, sei bei der Entscheidung nicht relevant gewesen. Bei objektiver Betrachtung erfüllten alle drei Kunstwerke die Voraussetzungen für eine Eintragung in das Verzeichnis national wertvollen Kulturgutes. Auch die Mehrheit der Mitglieder des Sachverständigenausschusses habe die Eintragung uneingeschränkt befürwortet.

Schließlich sei auch fraglich, ob der Kläger - die Rechtswidrigkeit der angegriffenen Verfügung unterstellt - überhaupt in eigenen Rechten verletzt sei. Das beklagte Land könne nicht beurteilen, ob der Kläger Eigentümer oder Verfügungsberech- tigter der Kunstwerke sei, weil ihm die Eigentums- und Besitzverhältnisse im Hinblick auf die drei eingetragenen Werke nicht bekannt seien.

Wegen der weiteren Einzelheiten des Sach- und Streitstandes wird ergänzend auf den Inhalt der Gerichtsakte sowie der vom beklagten Land vorgelegten Verwaltungsvorgänge Bezug genommen.

\section{Entscheidungsgründe}

Die Klage hat Erfolg. Sie ist zulässig und begründet.

Statthaft ist die Anfechtungsklage gemäß $\S 42$ Abs. 1 1. Alt. Verwaltungsgerichtsordnung (VwGO). Bei der Entscheidung über die Eintragung in das Verzeichnis national wertvollen Kulturgutes gemäß § 1 Abs. 1 Satz 1 KultgSchG handelt es sich um einen Verwaltungsakt (VG Hannover, Urteil vom 9.06.1989 - 6 A 69/87 - (Verwaltungsakt mit gleichsam dinglicher Kraft); OVG Niedersachsen, Urteil vom 19.05.1992 - 10 L 5248/91 -).

Dabei wirkt die Eintragung wegen ihrer konstitutiven Wirkung für und gegen jeden Verfügungsberechtigten (VG Berlin, Urteil vom 9.02.1994 - 1 A 29.92-).

Die Verfügungsberechtigung des Klägers ergibt sich daraus, dass die fraglichen Kunstwerke nach dem Vortrag der Beteiligten und in Ermangelung von substantiierten Hinweisen auf eine abweichende Rechtslage nach dem Tod von T3 der Mutter des Klägers - jedenfalls im Gesamthandseigentum der ungeteilten, aus dem Kläger und seiner Cousine 1. Grades - Frau T4 - bestehenden Erbengemeinschaft stehen. Ob der Kläger abweichend hiervon gemäß den von ihm gemachten Angaben sogar alleiniger Eigentümer des Bildes „T, 1935“ ist, bedarf hier keiner Klärung. Für die Annahme einer "Verfügungsberechtigung" im vorstehenden Sinne genügt es, Mitglied der Erbengemeinschaft zu sein, weil eine Verfügung über den Gegenstand gem. § 2040 BGB nur von den Erben gemeinschaftlich erfolgen kann.

Weiter kann es für die Frage der Statthaftigkeit der erhobenen Anfechtungsklage ebenso dahinstehen, ob die Eintragungsentscheidung gerade auch dem Kläger wirksam bekanntgegeben wurde, was insoweit erheblichen Zweifeln unterliegt, als nach dem Verwaltungsvorgang des beklagten Landes der als internationales Einschreiben an den Kläger nach Italien gesandte Brief unzugestellt zurückgesandt wurde (siehe das Retent am Ende von Bd. 5 der übersandten Verwaltungsvorgänge). Die Eintragungsentscheidung hat nämlich - nach dem Vorstehenden für jeden Verfügungsberechtigten - bereits dadurch äußere Wirksamkeit erlangt, dass sie durch inhaltsgleiche und am 3. März 2010 an den damaligen Verfahrensbevollmächtigten der Mutter des Klägers (den heutigen Prozessbevollmächtigten des Klägers) sowie an T4 zugestellte Bescheide bekanntgegeben wurde. Zwar ist die Mutter des Klägers noch vor dem 3. März 
2010 verstorben, allerdings wirkte die ihrem damaligen Verfahrensbevollmächtigten erteilte Vollmacht gemäß § 14 Abs. 21 . Hs VwVfG NRW über den Tod hinaus.

Aus der Verfügungsberechtigung des Klägers folgt seine gemäß § 42 Abs. 2 VwGO erforderliche Klagebefugnis hinsichtlich der drei betroffenen Kunstwerke. Dabei war der Kläger auch insoweit alleine zur Klageerhebung berechtigt, als die Kunstwerke im Gesamthandseigentum der aus dem Kläger und seiner Cousine bestehenden Erbengemeinschaft stehen. In der Anfechtung eines Verwaltungsaktes durch einen Miterben einer ungeteilten Erbengemeinschaft, der nach seiner Ansicht den Nachlass bzw. einzelne zum Nachlass gehörende Gegenstände rechtswidrig belastet, liegt eine sinngemäß dem § 2038 Abs. 1 Satz 2 Bürgerliches Gesetzbuch (BGB) entsprechende, den Nachlass schützende und deshalb „zur Erhaltung notwendige Maßregel", die jeder Miterbe ohne Mitwirkung der anderen treffen kann (BVerwG, Urteile vom 27.11.1981 - 4 C 1/81 -, NJW 1982, S. 1113 und vom 7. Mai 1965 - IV C 24.65 -, BVerwGE 21, 91ff.).

Ob die Cousine des Klägers mit der Klageerhebung einverstanden war, ist unerheblich, denn § 2038 Abs. 1 S. 2 Hs. 2 BGB räumt dem handelnden Miterben ein alleiniges, von der Mitwirkung bzw. Zustimmung der anderen Miterben unabhängiges Verwaltungsrecht ein (OVG NRW, Urteil vom 15.03.2011 - $20 \mathrm{~A}$ 2148/09-).

Schließlich wurde die Klage auch vor Eintritt der Bestandskraft der angegriffenen Entscheidung vom 26. Februar 2010 erhoben. Unbeschadet der Frage, ob für den Kläger überhaupt die einmonatige Klagefrist des $\S 74$ Abs. 1 Satz 1 VwGO lief, ist die Klageerhebung am 6. April 2010 innerhalb dieser Frist erfolgt. Gemäß § 57 Abs. 2 VwGO iVm § 222 Abs. 1 und 2 Zivilprozessordnung (ZPO) und $\S 188$ Abs. 2 BGB endete die durch die am 3. März 2010 erfolgten Zustellungen an die Mutter und die Cousine des Klägers ausgelöste Frist mit Ablauf des 6. April 2010. Dies ist der auf Ostermontag, den 5. April 2010, (und das vorausgehende Wochenende) nachfolgende Werktag.

Von einer Beiladung von Frau T4 hat die Kammer abgesehen. Es liegt kein Fall einer notwendigen Beiladung nach $§ 65$ Abs. 2 VwGO vor. Nach dieser Vorschrift sind Dritte beizuladen, wenn sie an dem streitigen Rechtsverhältnis derart beteiligt sind, dass die Entscheidung auch ihnen gegenüber nur einheitlich ergehen kann. Hierfür ist es nicht ausreichend, wenn eine einheitliche Entscheidung nur angesichts der tatsächlichen Verhältnisse oder logisch notwendig erscheint; es genügt nicht, wenn die betroffenen Rechte - wie hier - aufgrund des materiellen Rechts unmittelbar zusammenhängen (Vgl. Kopp/Schenke, VwGO, 16. Auflage, Rdnr. 15 für Fälle der Rechtsgemeinschaft/ Miterben sowie Rdnr. 17b).

Zur vergleichbaren Klage eines Mitgliedes einer ungeteilten Erbengemeinschaft gegen die vermögensrechtliche Restitution eines Nachlassgegenstandes an einen Dritten hat das Bundesverwaltungsgericht ausgeführt: „Zwar steht die Verwaltung des Nachlasses nach $\S 2038$ Abs. 1 S. 1 BGB den Erben gemeinschaftlich zu. Die zur Erhaltung des Nachlasses notwendigen Maßregeln, zu denen auch die hier erhobene Anfechtungsklage gehört, kann jedoch nach § 2038 Abs. 1 S. 2 Hs. 2 BGB jeder Miterbe ohne Mitwirkung der anderen treffen. Daraus folgt, dass die nicht klagenden Miterben weder als Streitgenossen, noch als Beigeladene am Verfahren zu beteiligen sind. Die sie betreffenden Auswirkungen des in einem solchen Prozess ergangenen Urteils bestimmen sich vielmehr unabhängig von ihrer Beteiligung nach dem Umfang der Rechtsstellung, mit der das bürgerliche Recht den klagenden Erben ausstattet." (BVerwG, Beschluss vom 20.10.1997 - 7 B 248/97 -).

Dem schließt sich die Kammer an.

In der Sache hat die Klage Erfolg. Die Entscheidung des Ministerpräsidenten des Landes Nordrhein-Westfalen vom 26. Februar 2010 über die Eintragung der fraglichen Kunstwerke in das Verzeichnis national wertvollen Kulturgutes ist formell rechtswidrig und verletzt den Kläger in seinen Rechten, § 113 Abs. 1 Satz 1 VwGO. Das Land Nordrhein-Westfalen besaß nicht die Verbandskompetenz zum Erlass der angegriffenen Entscheidung nach § 1 Abs. 1 Satz 1 KultgSchG. Dieser Mangel der Verbandskompetenz - der nicht zur Nichtigkeit der Eintragungsentscheidung führt - ist auch nicht nach § 46 VwVfG NRW unbeachtlich.

Gemäß § 1 Abs. 1 Satz 1 KultgSchG werden u.a. Kunstwerke, deren Abwanderung aus dem Geltungsbereich des Gesetzes einen wesentlichen Verlust für den deutschen Kulturbesitz bedeuten würde, in dem Land, in den sie sich bei Inkrafttreten des Gesetzes befinden, in ein „Verzeichnis national wertvollen Kulturgutes" eingetragen. Neben der Ermächtigung zur Eintragung von Kunstwerken und anderem Kulturgut in ein "Verzeichnis national wertvollen Kulturgutes" enthält die genannte Bestimmung gleichzeitig eine Zuständigkeitsregelung, die - da die Zuständigkeit verschiedener Bundesländer bei der Ausführung eines Bundesgesetzes als eigene Angelegenheit gemäß Art. 83, 84 GG untereinander abgegrenzt wird - die Verbandskompetenz betrifft (Vgl. zur Verbandskompetenz etwa Oldiges, DÖV 1989, S. 873 sowie OVG NRW, Urteil vom 3. Oktober 1978 - XV A 1927/75 -, NJW 1979, 1057ff).

Zuständig für die Entscheidung über die Eintragung ist nach dieser Regelung das Land dort gemäß § 2 Abs. 1 KultgSchG die oberste Landesbehörde, in dem sich das Kunstwerk bei Inkrafttreten des Kulturschutzgesetzes befand (ebenso Pieroth/Kampmann, NJW 1990, S. 1385 (1388)).

Danach besaß das beklagte Land nicht die Verbandskompetenz für die angegriffene Entscheidung. Nach den weder von dem beklagten Land bestrittenen, noch aus anderen Gründen zweifelhaften Angaben des Klägers befanden sich die fraglichen drei Kunstwerke sowohl zum Zeitpunkt des Inkrafttretens des Kulturschutzgesetzes vom 6. August 1955 (BGBI. I. S. 501) als 
auch zum Zeitpunkt der Verkündung der Neubekanntmachung des Kulturschutzgesetzes vom 8. Juli 1999 (BGBI. I, S. 1754) in Baden-Württemberg und nicht in Nordrhein-Westfalen.

Die demgegenüber vom beklagten Land eingenommene Rechtsauffassung, wonach dasjenige Bundesland für die Entscheidung nach § 1 Abs. 1 Satz 1 KultgSchG zuständig sein soll, in dem sich das fragliche Kulturgut zum Zeitpunkt der Einleitung des Unterschutzstellungsverfahrens befindet, überzeugt nicht. Entgegen der Einschätzung des beklagten Landes kann nicht davon ausgegangen werden, dass der Gesetzgeber durch die in § 1 Abs. 1 Satz 1 KultgSchG gewählte Formulierung nur seine Erwartung zum Ausdruck bringen wollte, dass eine Eintragung wertvollen Kulturgutes zeitnah und nicht erst dann erfolgt, wenn die Abwanderung droht (so aber VG Berlin, Urteil vom 29.11.2006 - 1 A $162.05-$ ).

Einer solchen Erwartung hätte der Gesetzgeber leicht durch andere, wesentlich eindeutigere Begriffe Ausdruck verleihen können, wie etwa „alsbald“ oder „unverzüglich“. Neben dem somit deutlich für eine Zuständigkeitsregelung sprechenden Wortlaut von § 1 Abs. 1 Satz 1 KultgSchG, vgl. VG Dresden, Urteil vom 5.11.2008 - 5 K 1837/05 -, spricht zudem ein Vergleich mit weiteren Vorschriften des Kulturschutzgesetzes gegen die Auffassung des beklagten Landes.

Anders als in § 1 Abs. 1 Satz 1 KultgSchG hat der Gesetzgeber in $\S 9$ Abs. 3 KultgSchG für bereits eingetragenes Kulturgut einem nachträglichen, nicht nur vorübergehenden Belegenheitswechsel ausdrücklich Bedeutung beigemessen. Zudem zeigt § 8 KultgSchG, dass dem Gesetzgeber die Möglichkeit, für die Frage der Zuständigkeit an den jeweiligen aktuellen Aufenthaltsort des Kulturgutes anzuknüpfen, durchaus bewusst war.

Weiterhin kann unbeschadet der Frage, ob nicht allein schon der klare Wortlaut des $\S 1$ Abs. 1 Satz 1 KultgSchG einem solchen Ergebnis entgegenstünde, auch nicht davon ausgegangen werden, dass der Zweck des Kulturschutzgesetzes, die Abwanderung national wertvollen Kulturgutes effektiv zu verhindern, zwingend eine Zuständigkeit des Bundeslandes der aktuellen Belegenheit erfordert. Im Gegenteil dürfte sich auch bei der Maßgeblichkeit der Belegenheit an einem in der Vergangenheit liegenden Stichtag durchaus ein effektiver Abwanderungsschutz erreichen lassen (vgl. VG Dresden, Urteil vom 5.11.2008 - 5 K 1837/05 -, wonach für den dort entschiedenen Fall der Schutzzweck des Kulturschutzgesetzes ausdrücklich die Einhaltung des Wortlauts der Zuständigkeitsregelung des § 1 Abs. 1 KultgSchG erfordere).

Durch das Abstellen auf einen in der Vergangenheit liegenden Zeitpunkt wird verhindert, dass ein befürchtetes bzw. bevorstehendes Unterschutzstellungsverfahren durch gezielte Ortswechsel unterminiert werden kann. Gleichzeitig wird so vermieden, dass nach einem möglicherweise unerwarteten Belegenheitswechsel ein anderes Bundesland überraschend zuständig wird und angesichts der unvorhergesehenen Situa- tion möglicherweise nicht schnell genug zu einer belastbaren Einschätzung der Bedeutung des Kulturgutes gelangt. Demgegenüber dürfte davon ausgegangen werden können, das gerade bei Kunstwerken, die wegen Ihrer Bedeutung für eine Eintragung in Betracht kommen, der jeweilige Aufenthaltsort jedenfalls für die Zeit nach dem 2. Weltkrieg so gut dokumentiert ist, dass eine verlässliche Bestimmung des Aufenthaltsortes zu einem bestimmten Stichtag regelmäßig unproblematisch möglich ist.

Schließlich ist zu berücksichtigen, dass der Gesetzgeber den Wortlaut von § 1 Abs. 1 Satz 1 KultgSchG bei der Neubekanntmachung des Kulturschutzgesetzes vom 8. Juli 1999 (BGBI. I, S. 1754) nicht abgeändert hat, obwohl zu diesem Zeitpunkt in der Literatur (vgl. Pieroth/Kampmann, NJW 1990, S. 1385 (1388)bereits die von der Kammer eingenommene, dem Wortlaut der fraglichen Regelung folgende Auffassung vertreten wurde.

Demgegenüber ergeben sich aus den Empfehlungen der Kultusministerkonferenz zum Vollzug des Kulturschutzgesetzes keine weiteren Erkenntnisse für die Frage der Zuständigkeit. Zwar mag es sein, dass diese grundsätzlich auch zur Auslegung des Kulturschutzgesetzes herangezogen werden können (VG Hannover, Urteil vom 9. Juni 1989 - 6 A 69/87 -).

Hier führt dies aber nicht weiter. Denn ohne, dass sich die maßgebliche Gesetzesfassung geändert hätte oder sonst ein nachvollziehbarer Grund für die Änderung der Empfehlungen ersichtlich wäre, weisen die Beschlüsse der Kultusministerkonferenz in verschiedene Richtungen. Zum einen heißt es im nicht mehr gültigen Beschluss der Kultusministerkonferenz vom 22. April 2004 am Ende: „Die Aussage in § 1 Abs. 1 des Gesetzes darüber, in welches Landesverzeichnis ein Kulturgut einzutragen ist, ist als Zuständigkeitsregelung, nicht als zeitliche Sperre zu verstehen. In das Verzeichnis kann auch Kulturgut eingetragen werden, das nach dem Inkrafttreten des Gesetzes in dessen Geltungsbereich gelangt." Zum anderen lautet der aktuelle, den vorgenannten Beschluss ersetzende Beschluss der Kultusministerkonferenz vom 29. April 2010 unter lit. D): „Die Regelungen in $\S 1$ Abs. 1 und § 10 Abs. 1 KultgSchG sind dahingehend zu verstehen, dass das Land für die Eintragung zuständig ist, in dem sich das Kultur- oder Archivgut zum Zeitpunkt der Einleitung des Verfahrens befindet. Ist der Aufbewahrungs- oder Standort des Kultur- oder Archivgutes nicht bekannt, richtet sich die örtliche Zuständigkeit nach dem Geschäfts- oder Wohnsitz des Eigentümers oder Besitzers. In die Verzeichnisse können auch Kultur- oder Archivgut eingetragen werden, die nach dem Inkrafttreten des Gesetzes in dessen Geltungsbereich gelangen oder gelangt sind."

Ob eine nicht nur zu einem vorübergehenden Zweck erfolgte Veränderung der Belegenheit, etwa in Anlehnung an $\S 9$ Abs. 3 KultgSchG, auch bei der nach § 1 Abs. 1 Satz 1 KultgSchG zu treffenden Entscheidung bedeutsam sein kann, so im Ergebnis VG Berlin, Urteil vom 29.11.2006 - 1 A 162.05 - für den dauerhaften Belegenheitswechsel eines Teiles der "Musikbibliothek 
Peters", bedarf hier angesichts der nur zur Versteigerung bei einem Ler Auktionshaus und damit zu einem vorübergehenden Zweck erfolgten Verbringung in das beklagte Land keiner Entscheidung. Ebenso kann hier offen bleiben, was hinsichtlich später geschaffenen oder eingeführten Kulturgutes gilt, vgl. hierzu etwa Bernsdorff/Klein-Tebbe, Kulturgutschutz in Deutschland, Teil A, § 1, Rdnr. 66, die insoweit § 1 Abs. 1 Satz 2 KultgSchG heranziehen, denn $\S 1$ Abs. 1 Satz 1 KultgSchG enthält eine für den vorliegenden Fall unmittelbar geltende Zuständigkeitsregelung.

Der Aufhebung der rechtswidrigen Entscheidung des Ministerpräsidenten des Landes Nordrhein-Westfalen vom 26. Februar 2010 steht auch nicht § 46 VwVfG NRW entgegen. Danach kann die Aufhebung eines Verwaltungsaktes, der nicht nach $\S 44$ VwVfG NRW nichtig ist, nicht allein deshalb beansprucht werden, weil er u.a. unter Verletzung von Vorschriften über die örtliche Zuständigkeit zustande gekommen ist, wenn offensichtlich ist, dass die Verletzung die Entscheidung in der Sache nicht beeinflusst hat.

Zwar führt die nach den vorstehenden Erwägungen nicht gegebene Verbandszuständigkeit des beklagten Landes nicht zur Nichtigkeit der angegriffenen Eintragungsentscheidung. Mangels Aufnahme in den Katalog des $§ 44$ Abs. 2 VwVfG NRW gibt es weder einen absoluten Nichtigkeitsgrund der fehlenden Verbandskompetenz noch ist die angegriffene Entscheidung des Ministerpräsidenten des beklagten Landes vom 26. Februar 2010 gemäß $\$ 44$ Abs. 1 VwVfG NRW nichtig. Insoweit fehlt es - auch wenn in der Regel bei wie hier fehlender Verbandskompetenz ein besonders schwerwiegender Fehler im Sinne der letztgenannten Vorschrift vorliegen dürfte - (vgl. Stelkens/ Bonk/Sachs, VwVfG, 6. Auflage, § 44 Rdnr. 159; Oldiges, DÖV 89, 873 (882)), jedenfalls an der nach § 44 Abs. 1 VwVfG NRW erforderlichen Offenkundigkeit des Mangels. Der Ministerpräsident des Landes Nordrhein-Westfalen ist nämlich im Rahmen der Zuständigkeitsregelung des $\S 1$ Abs. 1 Satz 1 KultgSchG grundsätzlich befugt, über die Eintragung von Kulturgut in das Verzeichnis national wertvollen Kulturgutes zu entscheiden. Der Zuständigkeitsmangel ergibt sich hier aus verschiedenen und im Einzelnen komplexen tatsächlichen und rechtlichen Gesichtspunkten des Einzelfalles und ist deshalb nicht offensichtlich. (Vgl. zu dem Fall, dass eine Behörde eines Bundeslandes bundesgesetzliche Verwaltungsangelegenheiten nach Art. 83 GG anstelle der zuständigen Behörde eines anderen Bundeslandes wahrnimmt Stelkens/Bonk/Sachs, VwVfG, 7. Auflage, § 44 Rdnr. 167; Kopp/Ramsauer VwVfG, 12. Aufl., § 44 Rdnr. $14 \mathrm{aE;}$ im Ergebnis ebenso OVG NRW, Urteil vom 3. Oktober 1978 - XV A 1927/75 - , NJW 1979, 1057ff.)

Allerdings stellt die fehlende Verbandskompetenz entgegen der Ansicht des beklagten Landes keine Verletzung von Vorschriften über die örtliche Zuständigkeit im Sinne des $\S 46$ VwVfG NRW dar (OVG NRW, Urteile vom 3.10.1978 - XV A 1927/75 - ,NJW 1979, 1057ff. sowie vom 24.11.1988 - 4 A 1526/87 -; Stelkens/Bonk/Sachs, VwVfG, 7. Auflage, § 46, Rdnr. 42; Kopp/
Ramsauer VwVfG, 12. Aufl., § 46, Rdnr. 23; Laubinger, VerwArchiv 1981, 333 (343); Oldiges, DÖV 89, 873 (882); i.E. wohl auch BVerwG, Urteil vom 10.12.1996 - 1 C 19/94 -, NVwZ-RR 1997, 388).

Bereits der Wortlaut des $\S 46$ VwVfG NRW ist eindeutig, indem er nur auf die Verletzung von Vorschriften über die örtliche Zuständigkeit Bezug nimmt. Die fehlende Verbandskompetenz kann auch nicht wie ein Mangel der örtlichen Zuständigkeit behandelt werden. Dem stehen die grundsätzlichen Unterschiede zwischen der Verbandskompetenz und der örtlichen Zuständigkeit entgegen. Während bei (bloß) fehlender örtlicher Zuständigkeit dennoch ein Verwaltungsrechtsverhältnis zum in der Sache kompetenten Rechtsträger besteht, erfolgt bei fehlender Verbandszuständigkeit ein nicht legitimierter Eingriff in fremde Verbandszuständigkeit. Damit steht der Anwendung des $\$ 46$ VwVfG NRW hier auch die fehlende Kompetenz des Landesgesetzgebers entgegen, einen Rechtsmangel, der die Verbandshoheit eines anderen Bundeslandes beeinträchtigt, einseitig durch eine Rechtsvorschrift des beeinträchtigenden Landes für unbeachtlich zu erklären (Oldiges, DÖV 89, 873 (882); OVG NRW, Urteil vom 24.11.1988 - 4 A 1526/87 -).

Schon aus diesen Gründen überzeugt die vom OVG Hamburg (OVG Hamburg, Urteile vom 16.02.1999 - Bf VI 2/97 - und vom 30.05.1996 - Bf VI(VII) 27/95 -) vertretene Auffassung nicht, jedenfalls in Fällen des Art. 83 GG sei § 46 VwVfG auch dann anwendbar, wenn die Verletzung örtlicher Zuständigkeiten zugleich eine Verletzung der Verbandskompetenz bedeute. Darüberhinaus begegnet gerade auch die in den genannten Entscheidungen des OVG Hamburg angestellte Erwägung Bedenken, im Verhältnis der Bundesländer untereinander komme der Einhaltung des Kompetenzbereichs bei der Ausführung von Bundesgesetzen gemäß Art. 83, 84 GG keine eigenständige, über den Mangel der örtlichen Zuständigkeit hinausreichende Bedeutung zu. Denn Art. 83 GG greift die verfassungsrechtliche Grundregel des Art. 30 GG auf, der zufolge die Ausübung aller staatlichen Befugnisse und die Erfüllung der staatlichen Aufgaben Sache der Länder ist, und bekräftigt sie im Sinne einer Verbandskompetenz jedes einzelnen Landes (Sachs, GrundgesetzKommentar, 6. Auflage, Art. 83 Rdnr. 3).

Danach kommt der verfassungsrechtlich geregelten Kompetenz der Länder zur Ausführung von Bundesgesetzen (u.a. dem Kulturschutzgesetz) als eigene Angelegenheit ein hoher Stellenwert zu. Sie ist mit einer weitreichenden Befugnis der Länder u.a. zur Einrichtung von Behörden und zur Regelung des Verwaltungsverfahrens verbunden und steht vergleichsweise schwach ausgeprägten Aufsichtsbefugnissen der Bundesregierung (Art. 84 Abs. 3 bis 5 GG) gegenüber. Dem würde es widersprechen, Eingriffe in diese Kompetenz unter den Voraussetzungen des $\S 46 \mathrm{VwVfG}$ (NRW) als unbeachtlich zu werten. Gerade bei der Ausführung des hier in Rede stehenden Kulturschutzgesetzes wird die eigene Stellung des ausführenden Länder dadurch betont, dass die Entscheidung über die Eintragung in das Verzeichnis national wertvollen Kulturgutes gemäß § 2 Abs. 2 Satz 1 KultgSchG obligatorisch die Beteiligung 
eines länderspezifischen Sachverständigenausschusses voraussetzt und im Ergebnis - auch wenn es sich um eine gebundene, gerichtlich uneingeschränkt überprüfbare Entscheidung handelt - (vgl. VGH Baden-Württemberg, Urteil vom 14.03.1986 - 5 S 1804/85), faktisch stark wertenden Charakters ist.

Diesem Ergebnis steht auch nicht das Urteil des Bundesverwaltungsgerichts vom 20. Februar 1992 entgegen, in dem das Bundesverwaltungsgericht die $\S 46$ VwVfG NRW inhaltlich entsprechende Vorschrift des $\S 42$ Satz 1 Sozialgesetzbuch Zehntes Buch (SGB X) auf die Änderung eines Ausbildungsförderungsbescheides durch ein nach Umzug des Klägers in ein anderes Bundesland nicht mehr zuständiges Amt für Ausbildungsförderung angewandt hat (BVerwG, Urteil vom 20.02.1992 - 5 C 66/88 -, BVerwGE 90, S. 25 - 37).

Zunächst kann bereits die Stellung der Länder bei Ausführung des Bundesausbildungsförderungsgesetzes im Auftrag des Bundes gemäß Art. 85 GG schon wegen der weitgehenden Weisungsbefugnisse und der sich auch auf die Zweckmäßigkeit erstreckenden Aufsicht des Bundes (Art. 85 Abs. 3 und 4 GG) nicht mit derjenigen bei der Ausführung von Bundesgesetzen als eigene Angelegenheit gemäß Art. 83, 84 GG verglichen werden. Zudem handelt es sich bei der Bestimmung des $§ 42$ SGB X um Bundesrecht. Aus diesem Grund steht der Anwendung dieser Vorschrift auf die fehlende Verbandskompetenz nicht das hinsichtlich der landesrechtlichen Vorschrift des § $46 \mathrm{VwVfG}$ NRW geltende Argument entgegen, dass dem Landesgesetzgeber die Kompetenz fehlt, die Verletzung fremder Verbandskompetenz durch eigenes Verfahrensrecht für im Ergebnis unbeachtlich zu erklären.

Schließlich ergibt sich auch aus dem Hinweis des Vertreters des beklagten Landes in der mündlichen Verhandlung auf gleichlautende Regelungen in anderen Landesverwaltungsverfahrensgesetzen nichts Abweichendes. Es ist ein rein faktischer und nach dem föderalistischen Prinzip nicht zwingender Befund, dass $\S 46$ Verwaltungsverfahrensgesetz für Baden-
Württemberg mit $\S 46 \mathrm{VwVfG} \mathrm{NRW} \mathrm{übereinstimmt.} \mathrm{Weder} \mathrm{ist}$ diese Vorschrift hier anwendbar, noch lässt sich aus der Übereinstimmung der Regelungen die Rechtsfolge ableiten, dass Verletzungen der Verbandskompetenz durch das beklagte Land unbeachtlich sein sollen.

Die Berufung war gemäß §§ 124 Abs. 2 Nr. 3, 124a Abs. 1 Satz 1 VwGO zuzulassen, weil die Fragen der Zuständigkeit nach dem Kulturschutzgesetz und der Anwendbarkeit des $§ 46 \mathrm{VwV}$ fG NRW auf Fälle der fehlenden Verbandskompetenz von grundsätzlicher Bedeutung sind. (Entscheidung von der Redaktion bearbeitet.)

\section{Aktuelle Informationen zur zeitgenössischen Kunst finden Sie im Internet unter:}

\section{www.art-in.de}

www.art-in-berlin.de
Ein Service der

Kunstagentur Thomessen

Admiralstraße 15 10999 Berlin

030/61402312 Tel. $030 / 6158152$ Fax 